\title{
A spectrographic receiver for laser spectrometers
}

\section{Macey Sandford, Paul Lucey, Xiaoli Sun, Daniel Cremons}

Macey Sandford, Paul Lucey, Xiaoli Sun, Daniel Cremons, "A spectrographic receiver for laser spectrometers," Proc. SPIE 10780, Multispectral, Hyperspectral, and Ultraspectral Remote Sensing Technology, Techniques and Applications VII, 107800J (23 October 2018); doi: $10.1117 / 12.2324818$

SPIE Event: SPIE Asia-Pacific Remote Sensing, 2018, Honolulu, Hawaii, United States 


\title{
A spectrographic receiver for laser spectrometers
}

\author{
Macey Sandford ${ }^{1}$, Paul Lucey ${ }^{1}$, Xiaoli Sun ${ }^{2}$, Daniel Cremons ${ }^{2}$ \\ ${ }^{1}$ University of Hawai'i at Manoa, Honolulu HI $96822,{ }^{2}$ NASA Goddard Space Flight Center, \\ Greenbelt MD 20771
}

\begin{abstract}
Multiband LiDAR systems, which are typically single wavelength in transmission and reception, are becoming more applicable for scientific use. However, traditional LiDAR receivers do not scale well to tens or hundreds of received bands. We introduce the design for a spectrographic receiver using an array detector for laser spectrometers and present two of the many possible applications: fluorescence spectroscopy in the visible range and IR reflectance spectroscopy. Each laser pulse has the capability of exciting a target in various wavelengths, and a spectrographic receiver would be able to interpret this excitation, while a typical LiDAR consisting of single wavelength receiver would not. Using a spectrograph in a system with a pulsed laser in the visible or UV range is capable of the detection of fluorescent signal. These spectra reveal the presence of organics and is an applicable technology for planetary science. A spectrograph coupled with a pulsed laser in the IR range shows capability of detecting the presence of water in various forms also applicable technology for both Earth and planetary science. Both systems utilize a Czerny-Turner spectrograph design with a ZnSe prism for the dispersion of light onto an Avalanche Photo Diode (APD). This paper introduces the concept and design of a spectrographic receiver for laser spectrometers, as well as two possible applications.
\end{abstract}

Keywords: Spectrometer, instrumentation, remote sensing, fluorescence, infrared, lasers, planetary science

\section{INTRODUCTION}

Typical LiDAR systems operate using a single wavelength combined with a single wavelength receiver. Differential absorption LiDARs (DIAL) for gas detection use two wavelengths, and typically employ time multiplexed receivers or dichroic beam splitters to send the return beam to separate respective detectors. As technology progresses, multiband LiDAR systems are becoming more practical. This includes using many laser transmission bands to probe complex chemical targets or stimulating the target with a single laser frequency that results in multiband response such as standoff Raman or fluorescent LiDARs. These two types of multiband LiDARs stress existing detection techniques. Time multiplexing is only effective for a small number of bands if targets are variable over the timescale of the laser wavelength variation, and is not effective for LiDARs where single bands stimulate a spectral response. Dichroic beam splitter approaches are also limited in band count as losses at each wavelength division mount and the arrangement of detectors becomes challenging (Figure 1). Here, we present a design that simplifies the optical path and that is scalable; a spectrographic receiver and discussion of two applications of a spectrographic receiver for laser spectrometers concerning fluorescence and IR systems. Our concept incorporates a spectrographic receiver with a high-speed photodiode array (APD) (Figure 2). The spectrographic receiver is based on the Czerny Turner spectrograph design. The applications are a laser fluorescence spectrometer with a single transmitting wavelength, requiring receiving technology for many tens or hundreds of receiving wavelengths, and an IR multiband laser reflectance system using 6 transmitting wavelengths and 6 receiving wavelengths (Figure 3).

The spectrograph concept is scalable to any wavelength range within limits. In this paper, the spectral component of the spectrograph discussed covers the visible range from 0.55 to 0.75 microns and in the IR range from 1.5 to 3.6 microns. The visible range aims to conduct fluorescence spectroscopy, where organics have a strong signal. The IR range aims to conduct laser reflectance spectroscopy of various forms of water and organics. With this spectral sensitivity combined with the high sensitivity of the APD detectors discussed, we present a spectrographic reviver enabling a wide range of remote sensing studies in the visible and IR wavelength ranges. 


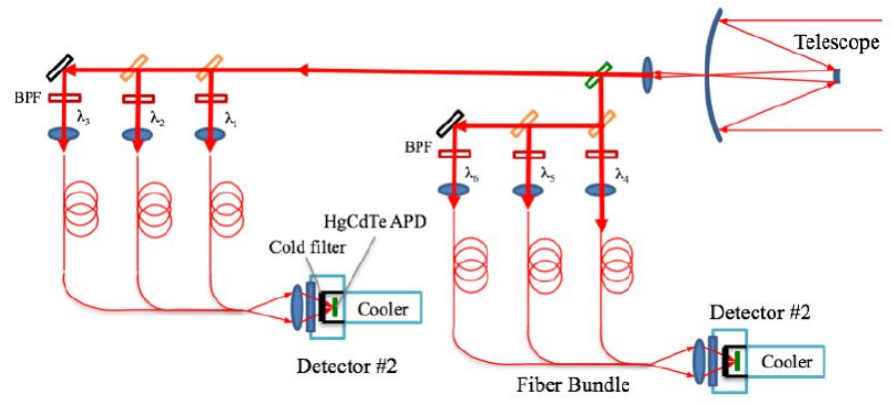

Figure 1: Block diagram of a multiband IR LiDAR receiver using multiple dichroic beam splitters and detectors illustrating the complexity of the concept. Losses are experienced at each dichroic and at fiber couplings. In this example, two wavelength ranges required two separate detectors.

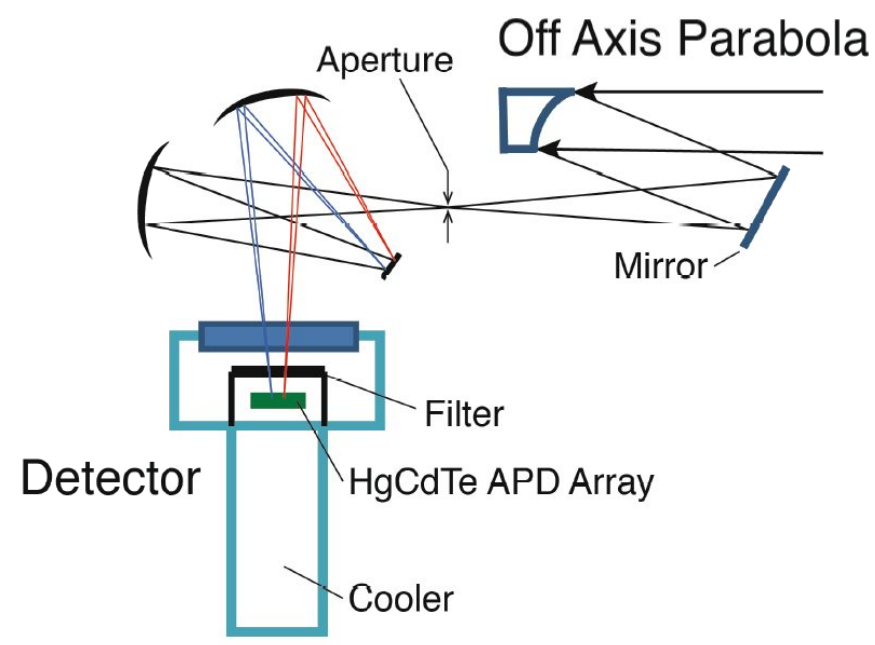

Figure 2: Schematic of proposed receiver concept, including laboratory off axis parabola for light collection. Note the compactness and simplicity of the concept compared to the current scheme (Figure 1).

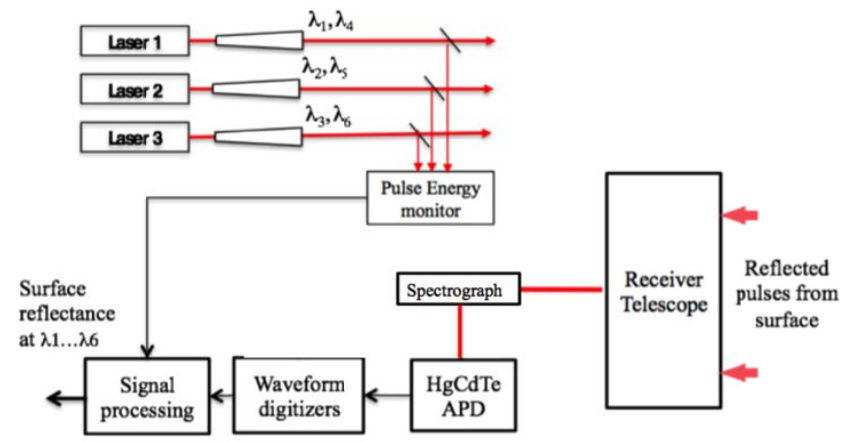

Figure 3: Generalized schematic of the IR spectral LiDAR. In this depiction three 2-band lasers transmit boresighted energy to the surface. The receiver telescope focuses the return signal on the aperture of the spectrograph that disperses the light in wavelength on the APD array. The waveforms are digitized and sent to signal processing to extract range and the integrated intensity of the return pulse. Meanwhile, intensity and timing of each outgoing pulse is measured by the pulse energy monitor and fed to signal processing. This project focusses on the spectrograph and APD detectors. 


\section{METHODS}

In this section, we discuss the design and two possible applications. A LiDAR system consists of a laser transmitter, a receiver, and time-resolved electronics that determine the range of the target using timing electronics and the returned pulse characteristics. Our design describes the implementation of a spectrographic receiver, capable of ranging and detection of various wavelengths simultaneously. The key to the spectrographic design is the use of array detectors, and a ranging LiDAR requires high-speed detectors. Avalanche photodiode arrays are available both in the visible and IR and will be described below. Relevant IR lasers are available or in development (Sun et al. 2014; Li et al. 2017; Hayne et al. 2013). This design reduces the optical path length and preserves intensity as compared to the IR multiband LiDAR shown in Figure 1, which uses both multiple beam splitters and time-separated pulses to collect data. The spectrographic receiver approach is scalable and applicable to several types of laser induced spectroscopy systems. Hence, a single receiver development can be applied to multiple instruments, including discrete wavelength transmitters as well as broadband transmitters or fluorescent LiDARs. As an additional benefit, in a discrete laser wavelength system, the nominally unilluminated pixels on the detector provide a measure of the instrument's stray light and general system background. The measurement of background yields a powerful and extremely sensitive passive spectral measurement system given the near photon counting nature of the detectors. For example, in passive mode the Lunar Orbiter Laser Altimeter single band detector was used for photometric studies (Barker et al. 2016) and in searches for lofted dust in the lunar atmosphere (Smith et al. 2016). The LIBS laser spectrometer on the Mars Science Laboratory has employed this method for passive spectroscopy in the visible portion of the spectrum (Johnson et al. 2015; Lucey et al. 2017).

\subsection{Spectrograph}

A Czerny-Turner spectrograph comprises two spherical mirrors with a flat diffraction grating. By folding the beam with the two mirrors, the spectrograph is compact. Figure 4 shows a ray trace of our design that features $>80 \%$ ensquared energy within the 64 micron square IR pixels at all wavelengths and $>95 \%$ within the 300 micron visible APD detectors at all wavelengths.

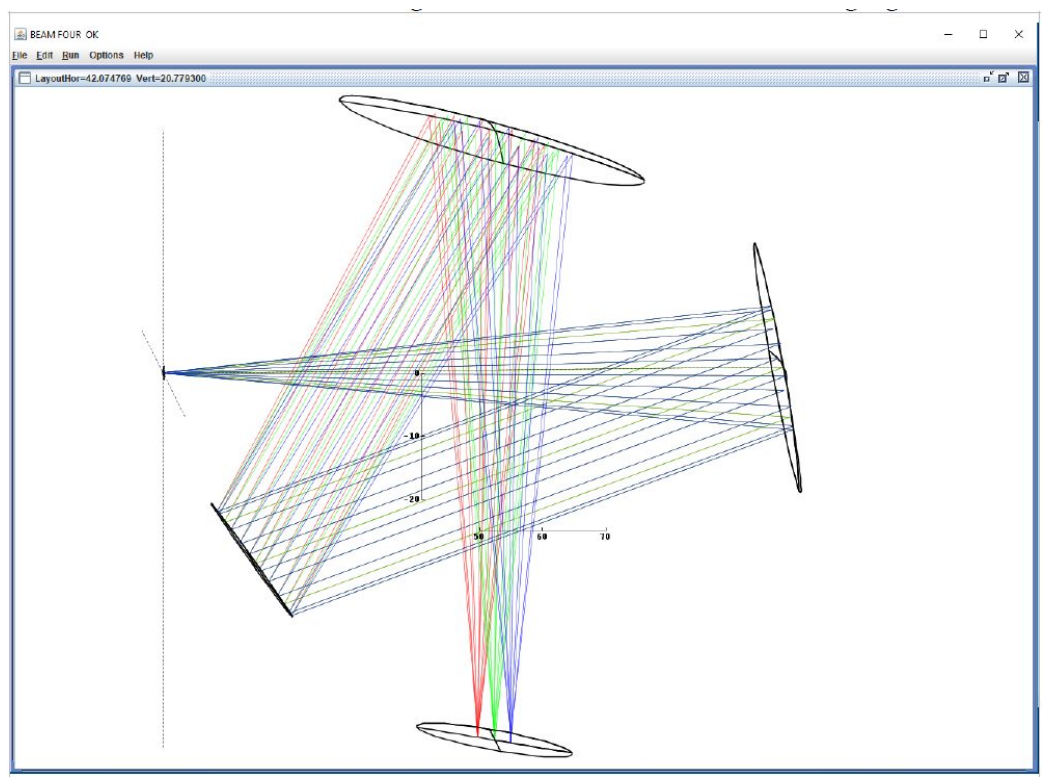

Figure 4: Ray trace of Czerny-Turner spectrograph for visible fluorescence spectroscopy. The overall size of the instrument is not optimized, current it is $100 \times 100 \times 50 \mathrm{~mm}$, but conveys the concept. In this design the ensquared energy is over $95 \%$ for the 300 micron visible APD pixels.

In the visible version, the spectrograph design operates within the range of 0.55 to $0.75 \mu \mathrm{m}$. This range sufficiently captures the fluorescence signal from most organics as well as fluorescent minerals using a $530 \mathrm{~nm}$ laser transmitter. The spectrograph is designed for a $7.5 \mathrm{~mm} 25$ element linear detector array (Section 2.3) resulting in a spectral sampling of 8 
$\mathrm{nm}$. In order to filter out the incident $532 \mathrm{~nm}$ laser light, a long-pass filter is placed in front of the spectrograph. The fluorescent return signal can distinguish organics and non-organics based on the properties on short-lived versus longlived fluorescence (Misra et al. 2016).

The IR spectrograph is similar to the visible spectrograph, but includes additional features due to the properties of the spectral range. Since IR receivers are sensitive to thermal background detection, a cold filter in the detector rejects thermal background beyond 3.5 microns. Order separation is also required, given the breadth of the spectral range discussed. In this application, we take advantage of a two-row linear detector array and execute cross dispersion using a $\mathrm{ZnSe}$ prism. The prism separates two adjacent spectral orders, with spatial projection in agreement with the size of the detector. For the first order, $\mathrm{m}=1$, the spectra projects from 2.5 to 5 microns on one row of the detector and for the second order, $\mathrm{m}=2$, the spectra projects from 1.25 to 2.5 microns on the second row of the detector. Since the prism is weak, the curvature of the spectrum is negligible. The dispersion in the IR spectrographic receiver is designed to place 2.4 to 3.6 microns on a 30-pixel detector, resulting in a $40 \mathrm{~nm}$ spectral resolution in the first order and a $20 \mathrm{~nm}$ spectral resolution for the second order, since the 30 pixels cover a smaller range (1.25 to 1.8 microns). In all, the properties of the spectrograph vary slightly from one spectrographic receiver to the other but the concept is scalable.

\subsection{Passive backgrounds}

Since the IR instrument concept collects reflected and emitted radiance, there is a contribution of this radiance to the total signal detected. In the IR, the ambient solar background signal is much smaller than the laser signal during the brief duration of the laser pulse, even at solar noon, but it is not zero. Two methods are available to estimate and remove the lunar background. The first is to sample the signal before and after the arrival of the laser pulse, though this introduces the uncertainty associated with downtrack variation in radiance, which can be large (e.g. at the edges of shadows). However, in the discrete wavelength IR case, since the lasers only illuminate a small portion of the detector array, the passive signal can be interpolated across the laser-occupied pixels. Ambient radiance is more problematic for the fluorescent mode as the fluorescent spectrum is continuous, can be weak, and in the case of mineral fluorescence longlived (milliseconds). In this case, temporal sampling may be required or limiting operation to lower radiance backgrounds, especially at night.

\subsection{Lasers}

Figure 5 shows the Quantel VIRON compact solid-state laser, used for the application of the spectrographic receiver for fluorescence spectroscopy. It is a compact active Q-switched solid-state pulsed laser. The laser utilizes a diode to generate the lasing photons. Its capabilities include a $20 \mathrm{~Hz}$ operating rate, conductive cooling, $15 \mathrm{~mJ} / \mathrm{pulse}$ pulse energy, and a $10 \mathrm{~ns}$ pulse width. The IR instrument uses a diode pumped monolithic IOPO (Intracavity Optical Parametric Oscillator) laser, developed at GSFC, to generate laser pulses in the wavelengths specified (Wang et al. 2008; Fahey et al. 2016) (Figure 6).

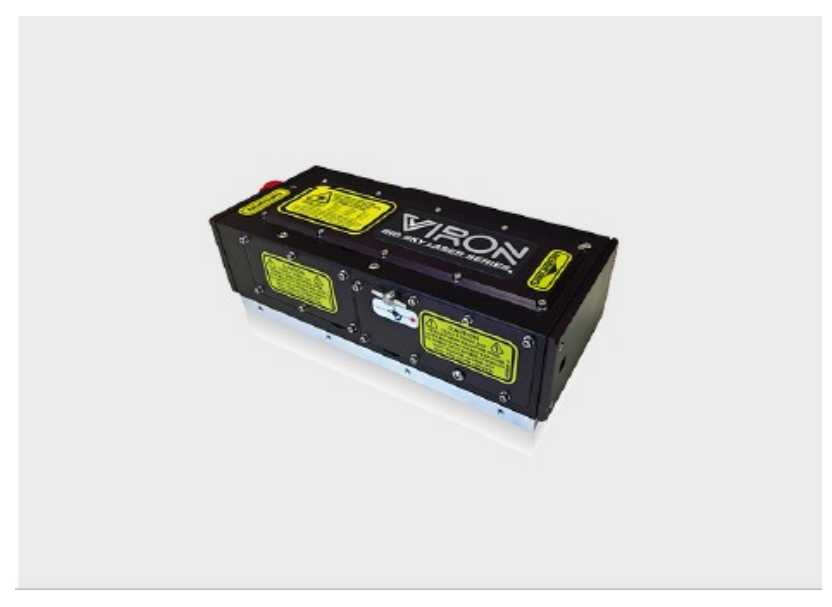

Figure 5: Viron pulsed $532 \mathrm{~nm}$ laser for use in the visible instrument. 


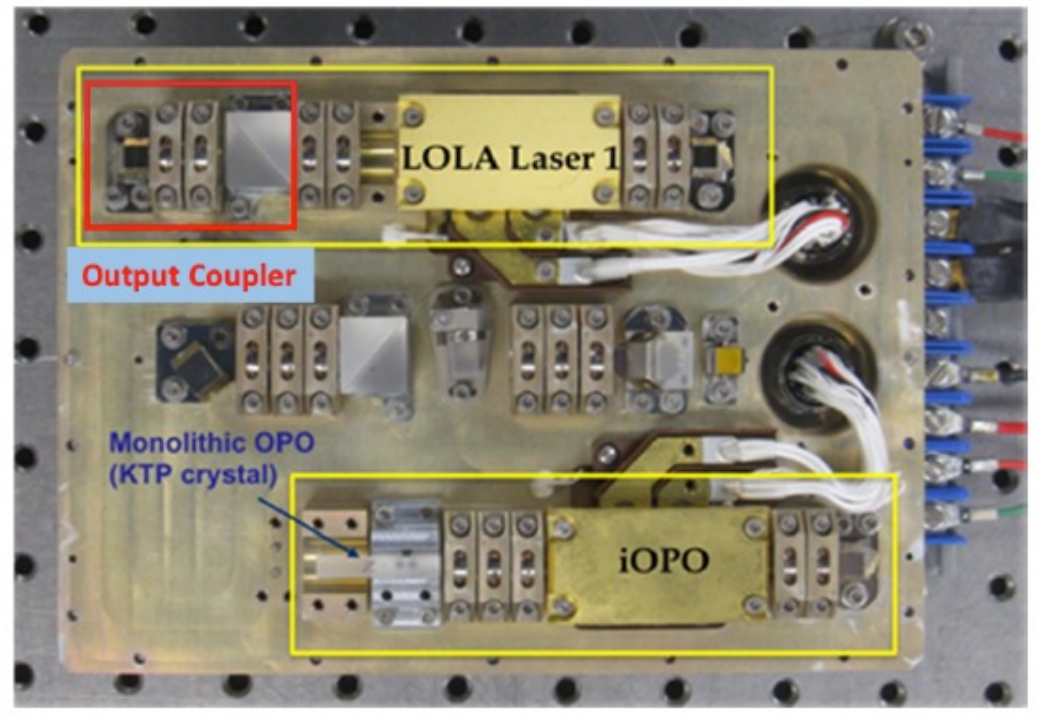

Figure 6: IOPO laser (bottom) in comparison to the LOLA laser (top). The IOPO laser is almost identical to the LOLA laser except for the wavelength conversion section.

\subsection{Detectors}

The instrument operating in the visible range would use a commercial Si APD array by Excelitas and is shown in Figure 7. The detector array consists of $25300 \mu \mathrm{m}$ APDs. The excitation wavelength of the laser is $532 \mathrm{~nm}$ and fluorescence occurs at longer wavelengths than the source wavelength, so it will not detect light from higher orders of the dispersed light. As stated earlier, the laser light is excluded using a long-pass filter.

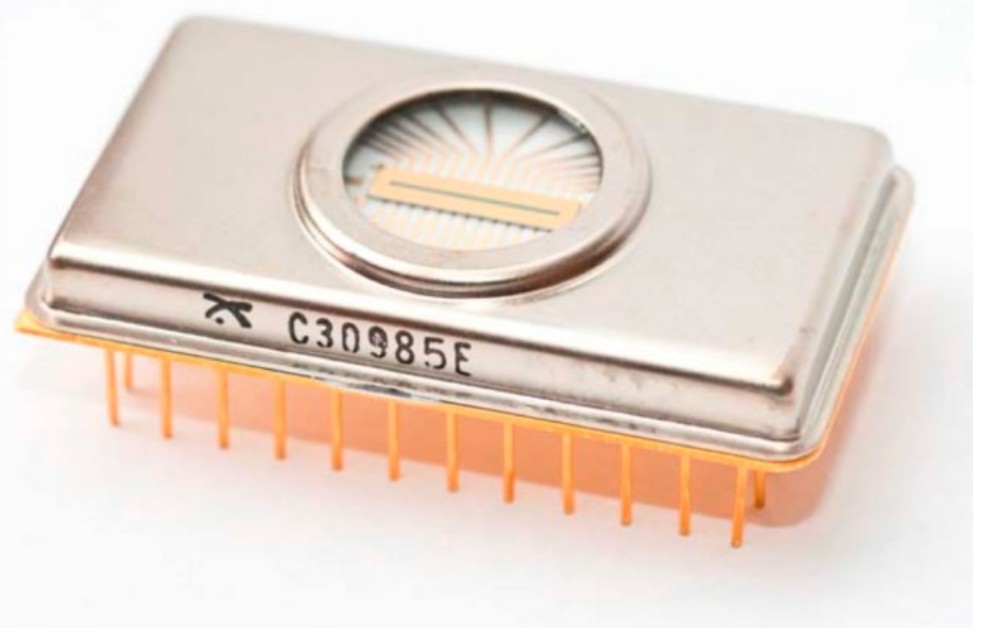

Figure 7: Excelitas 25 element Si APD to be used for the visible testing.

The IR instrument uses a 2x8 pixel HgCdTe APD array, from GSFC and DRS as shown in Figure 8 in an LN2 dewar. These detectors have a quantum efficiency $>90 \%$ from the visible to 5 micron range. The read-out integrated circuits (ROIC) operate at cryogenic temperatures, allowing the photo-multiplication gain of the APD to sufficiently override the noise associated with the ROIC. There is little noise from the photoelectron multiplication process associated with the APD. In Figure 9, we display the spectral response of the HgCdTe APD (Beck et al 2006; Sun et al. 2015). This graphic shows the detectors approaching a quantum-limited performance before reaching the maximum 
APD gain and the SNR is nearly constant at higher APD gain ("noiseless gain"). The detectors discussed were for $\mathrm{CO}_{2}$ and $\mathrm{CH}_{4}$ LiDAR measurements in 2014, 2015, and 2016 (e.g. Sun et al. 2016). They demonstrated unprecedented sensitivity and linear dynamic range.

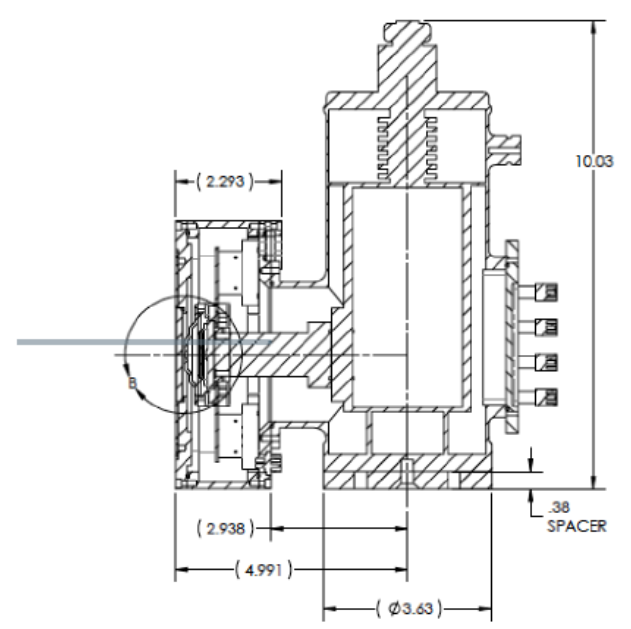

Figure 8: Mechanical drawing of the DRS HgCdTe detector array in an LN2 dewar.

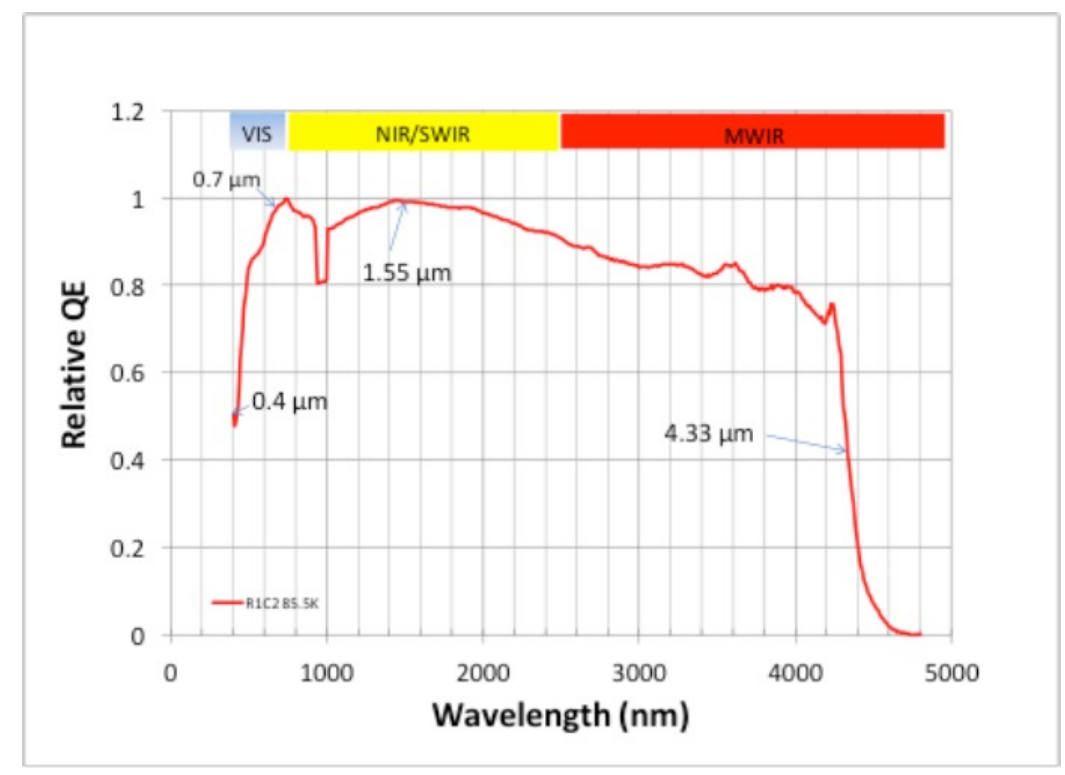

Figure 9: Spectral response of the HgCdTe APD. The detector has a $>80 \%$ quantum efficiency from visible to $4.3 \mu \mathrm{m}$, covering all the wavelengths of the proposed multi-wavelength IR LiDAR.

\section{IMPACT \& APPLICATIONS}

This spectrographic receiver, shown in Figure 11, enables various spectroscopic LiDAR instruments that detect active reflectance and spectral fluorescence LiDARs. Issues with current LiDARs are mitigated with the proposed instrument design and functionality. These issues include noise associated with less known range, temporal multiplexing of spectral LiDAR returns, and the reduction of sensitive passive background measurements, due to its low-light-level 
operation. In all, this instrument would function as a universal tool for laser reluctance and fluorescence spectrometers in the future.

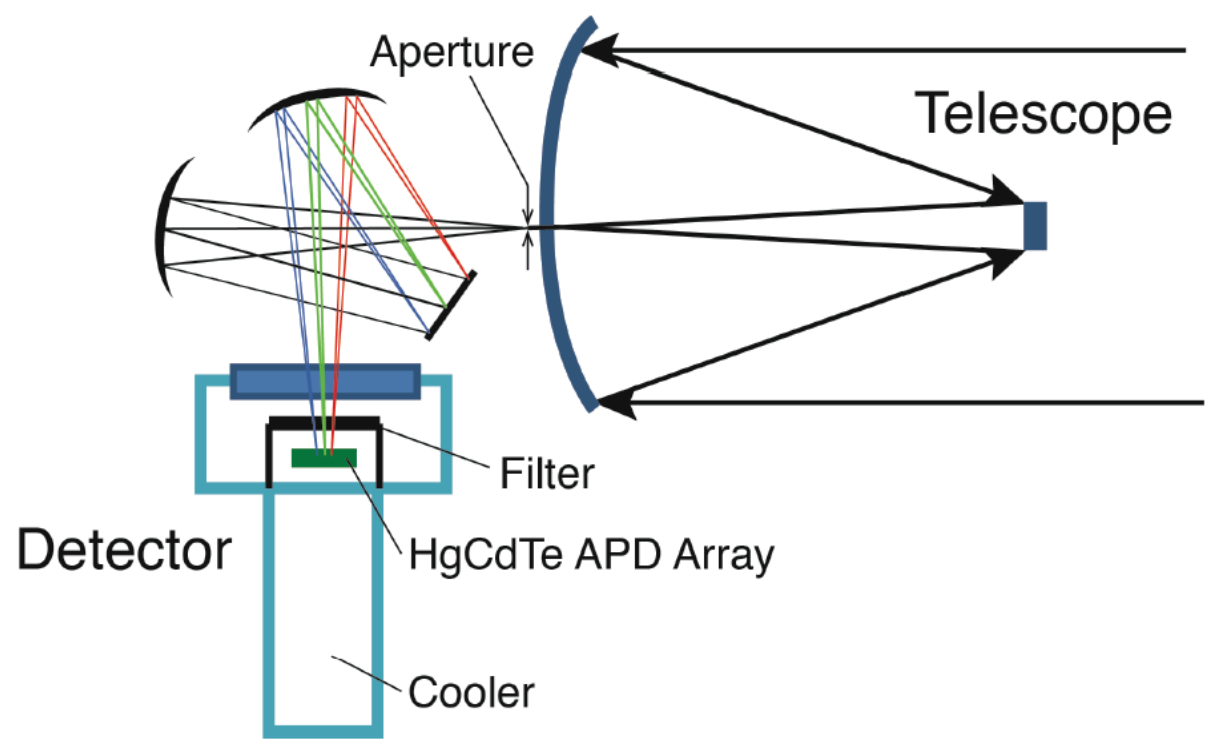

Figure 11: Block diagram of the field configuration of the proposed IR spectrographic LiDAR receiver.

\section{CONCLUSION}

As LiDAR applications increase to include spectrally complex targets stimulated by laser radiation, either reflection from multiband sources or fluorescence or Raman from single band sources, traditional time multiplex or dichroic receivers are either not applicable or become impractically complex. A spectrographic approach using a grating spectrograph can receive all wavelengths with a relatively simple optical system, using array detectors such as avalanche photodiodes. Avalanche photodiode arrays are available in both the IR and visible portions of the spectrum, enabling a wide range of multiband LiDAR applications when coupled with a spectrograph. In this paper, we described the application of a spectrographic approach to an IR multiband laser transmitter LiDAR, and a fluorescence spectroscopy LiDAR using a single laser transmitter. The proposed spectrographic LiDAR receiver is applicable to a wide range of missions and laser transmitter modalities. It enables measurement of range, of value to any mission, but also removes the significant source of noise inherent in range variation. It also allows simultaneous detection of all wavelengths, mitigating noise due to surface reflectance variations.

\section{REFERENCES}

[1] Alexander VV, Shi Z, Islam MN, Ke K, Kalinchenko G, Freeman MJ, Ifarraguerri A, Meola J, Absi A, Leonard J, Zadnik JA. Field trial of active remote sensing using a high-power short-wave infrared supercontinuum laser. Applied optics. 2013 Sep 20;52(27):6813-23.

[2] Bandfield, J., Poston, M., Klima, R., \& Edwards, C. (2017). A prominent and ubiquitous oh/h2o feature in corrected lunar spectra. In Lunar and Planetary Science Conference. volume 48.

[3] Barker MK, Sun X, Mazarico E, Neumann GA, Zuber MT, Smith DE. Lunar phase function at $1064 \mathrm{~nm}$ from Lunar Orbiter Laser Altimeter passive and active radiometry. Icarus. $2016 \mathrm{Jul}$ 15;273:96-113.

[4] Clegg SM, Wiens RC, Maurice S, Gasnault O, Sharma SK, Misra AK, Newell R, Forni O, Lasue J, Anderson RB, Nowak-Lovato KL. Remote geochemical and mineralogical analysis with supercam for the Mars 2020 rover. InLunar and Planetary Science Conference 2015 Mar (Vol. 46, p. 2781).

[5] Cohen, B.A., Hayne, P.O., Greenhagen, B.T. and Paige, D.A., 2015, October. Lunar Flashlight: exploration and science at the moon with a 6U CubeSat. In Annual Meeting of the Lunar Exploration Analysis Group (Vol. 1863, p. 2008). 
[6] Farmer, F.H.; Brown, C.A., Jr.; Jarrett, O., Jr.; Campbell, J.W.; Staton, W.L. Remote Sensing ofPhytoplankton Density and Diversity in Narragansett Bay Using an Airborne Fluorosensor. In Proceedings of the 13th International Symposium for Remote Sensing of Environment, Ann Arbor, MI, USA, 23-27 April 1979. Remote Sens. 2013, 54420

[7] Hayne, P.O., Cohen, B.A., Sellar, R.G., Staehle, R., Toomarian, N. and Paige, D.A., 2013, October. Lunar flashlight: Mapping lunar surface volatiles using a cubesat. In Annual Meeting of the Lunar Exploration Analysis Group (Vol. 1748, p. 7045).

[8] Hoge, F.E.; Swift, R.N. Airborne simultaneous spectroscopic detection of laser-induced water Raman backscatter and fluorescence from chlorophyll a and other naturally occurring pigment. Appl. Opt. 1981, 20, 3197-3205.

[9] Johnson JR, Bell JF, Bender S, Blaney D, Cloutis E, DeFlores L, Ehlmann B, Gasnault O, Gondet B, Kinch K, Lemmon M. ChemCam passive reflectance spectroscopy of surface materials at the Curiosity landing site, Mars. Icarus. 2015 Mar 15;249:74-92.

[10]Li SX, Anthony WY, Sun X, Fahey ME, Numata K, Krainak MA. A multi-wavelength IR laser for space applications. InSPIE Defense+ Security 2017 May 5 (pp. 101910L-101910L). International Society for Optics and Photonics.

[11]Lucey, P. G.; Sun, X.; Li, S. X.; Numata, K.; Neumann, G. A.; Abshire, J. B.; Smith, D. E.,An Infrared Multiwavelength Lidar for Compositional Mapping, 3rd International Workshop on Instrumentation for Planetary Mission, held 24-27 October, 2016 in Pasadena, California. LPI Contribution No. 1980, id.4069 10/2016 LPI 2016LPICo1980.4069L

[12]Lucey, P. G.; Sun, X.; Petro, N.; Farrell, W.; Abshire, J. B.; Mazarico, E.; Neumann, G. A.; Green, R.; Thompson, D. E.; Greenberger, R.; Hurley, D.; McClanahan, T. P.; Smith, D. E.; Zuber, M. T., The Lunar Volatiles Orbiter: A Discovery Class Lunar Water Mission, Annual Meeting of the Lunar Exploration Analysis Group, held 1-3 November, 2016 in Columbia, Maryland. LPI Contribution No. 1960, id.5049, 11/2016 LPI 2016LPICo1960.5049L

[13] Lucey PG, Trang D, Johnson JR, Glotch TD. Derivation of optical constants for nanophase hematite and application to modeled abundances from in-situ Martian reflectance spectra. Icarus. 2018 Jan 15;300:167-73.

[14] Michael C. Storrie-Lombardi and Birgit Sattler. Astrobiology. September 2009, 9(7): 659-672. https://doi.org/10.1089/ast.2009.0351

[15] Smith DE, Zuber MT, Barker M, Mazarico E, Neumann GA, McClanahan TP, Sun X. An Experiment to Detect Lunar Horizon Glow with the Lunar Orbit Laser Altimeter Laser Ranging Telescope. InEGU General Assembly Conference Abstracts 2016 Apr (Vol. 18, p. 10596).

[16] Sun X, Abshire JB, Beck JD. HgCdTe e-APD detector arrays with single photon sensitivity for space lidar applications. InAdvanced Photon Counting Techniques VIII 2014 May 28 (Vol. 9114, p. 91140K). International Society for Optics and Photonics.

[17] Sun X, Abshire JB, Ramanathan AK, Mao J, Kawa SR. Receiver Performance Analysis of a Multi-wavelength Sampling Integrated Path Differential Absorption CO2 Lidar. InAGU Fall Meeting Abstracts 2016 Feb.

[18] Sun, X., J. B. Abshire, and J. Beck (2014), HgCdTe e-APD detector arrays with single photon sensitivity for space lidar applications, Proc. SPIE 9114.

[19] Vinckier Q, Crabtree K, Paine CG, Hayne PO, Sellar RG. Design and characterization of a low cost CubeSat multi-band optical receiver to map water ice on the lunar surface for the Lunar Flashlight mission. InInfrared Remote Sensing and Instrumentation XXV 2017 Aug 30 (Vol. 10403, p. 104030R). International Society for Optics and Photonics.

[20] Yang J, Gong W, Shi S, Du L, Zhu B, Sun J, Song S. Excitation Wavelength Analysis of Laser-Induced Fluorescence LiDAR for Identifying Plant Species. IEEE Geoscience and Remote Sensing Letters. 2016 Jul;13(7):977-81. 